\title{
THERMO-OPTICAL SPECTROSCOPY OF LOW TEMPERATURE SOLIDS
}

\author{
H. PARKER, K.W. HIPPS and A.H. FRANCIS * \\ Department of Chemistry, University of Michigan, Ann Arbor, Michigan 48109, USA
}

Received 6 December 1976

\begin{abstract}
A discussion of an experimental procedure for determining the absorption spectra of low temperature solids by thermooptical spectroscopy is presented. The method may be extended to the direct measurement in low temperature solids of radiationless relaxation dy namics. The development of a suitable detector utilizing thin films of semiconducting germanium in an amorphous state is discussed. Performance data for the detector are presented and the results of some measurements on crystalline samples of biacetyl and several inorganic semiconductors are illustrated and briefly discussed.
\end{abstract}

\section{Introduction}

Recently, there has been a revived interest in the technique of opto-acoustic spectroscopy. Opto-acoustic experiments were first performed by Robin [1] in the gas phase and later on solids by Harshbarger and Robin [2] and Rosencwaig [3]. The techniques employed for both solids and gases are closely related and are derived from the original experiments by Alexander Graham Bell [4] performed in 1880. The technique has proved extremely useful in obtaining both qualitative and quantitative information concerning the absorption spectra of solids, liquids and gases which, for a variety of reasons, was not available from conventional absorption or emission spectroscopy. Additionally, gas phase opto-acoustic spectroscopy has provided detailed information concerning the relative rates of radiationless relaxation and certain photochemical processes which occur in the higher excited states of molecules [5].

In opto-acoustic spectroscopy, heat produced by the radiationless relaxation processes which occur subsequent to electronic excitation of molecules in a gaseous sample or solid sample is detected as a pressure change in either the sample gas or a coupling gas, respectively. If the excitation intensity is modulated at

* Part of this work was completed in the Department of Chemistry, University of Illinois at Chicago Circle. acoustic frequencies, a microphone may be utilized as a convenient and sensitive pressure transducer. The magnitude of the heating effect and its time response are then related to the microphone signal amplitude and phase shift relative to the modulating waveform [6].

The magnitude of the heating effect is related to quantum yield via a particular set of radiationless relaxation pathways accessible at a particular excitation energy, while the phase shift is related to the "effective" relaxation rate via the particular set of pathways.

Recently, there has been some interest in translating the op to-acoustic experiment as performed in the vapor phase into a framework suitable for application in low temperature solid state spectroscopy $[7,8]$. Our effort was motivated by several interests, principal among which were the desire to compare and contrast radiationless relaxation dynamics in solids with those in the vapor phase and the anticipation of greater resolution afforded by solids at low temperatures.

\section{Experimental}

Our central task in the present work was the development of a thermal detector capable of measuring the extremely small temperature changes produced by raditionless relaxation and yet having a response time better than $100 \mathrm{kHz}$. Several types of detectors were 
investigated, among which the most extensively studied were superconducting metal films biased in the superconducting transition region [9], carbon film bolometers [10] and narrow band gap semiconductor films [11]. The superconducting bolometer detectors were prepared from tin-lead alloy evaporated films in order to broaden the superconducting transition region of the device. Samples were either placed in direct contact with the detector surface or connected thermally via a saphire heat pipe. Al though the sensitivity of the detector constructed in this manner is adequate for the measurement of the signal levels produced with only moderate irradiation levels, the dynamic range of the detector is extremely small due to the narrowness of the superconducting transition temperature region and the detector must be carefully biased in the middle of the operating range in order to function properly. Although this may be achieved with relative ease magnetically [12], the biasing requirement added considerably to the complexity of the experiment, and we sought a device which would provide comparable sensitivity without the necessity of magnetic of thermal biasing.

Detectors constructed using carbon ink [13], aglomerated [13] and evaporated [14] carbon and colloidal [15] graphite (aquadag) films proved to be considerably less sensitive than the superconducting detectors and, although the sensitivity may have been adequate for many of the experiments contemplated, the devices also proved to be excessively noisy electrically.
A suitable detector was fabricated from an amorphous semiconductor film. The semiconductor film is a partially disordered film approximately $1000 \AA$ thick of p-type germanium deposited by vacuum evaporation on a quartz substrate. When the substrate temperature is above approximately $500^{\circ} \mathrm{C}$ during the exaporation procedure, germanium films which are crystalline in nature and possess considerable long-range order are obtained [16]. These films have both a lower specific resistance and a smaller temperature coefficient of resistance than the amorphous films which are obtained when the substrate temperature is mantained below $300^{\circ} \mathrm{C}$ during the evaporation [17]. The amorphous material has short range positional order over two or three lattice spacings, but long-range order is absent as shown by $X$-ray and electron diffraction studies [18]. The high intrinsic resistance of the amorphous material produced detectors with operating resistances on the order of $10 \mathrm{M} \Omega$ at $2 \mathrm{~K}$, which resulted in electrical time constants too long for high frequency modulation. The purely crystalline material, on the other hand, produced with resistances on the order of $1 \mathrm{k} \Omega$ at $2 \mathrm{~K}$ but with. temperature coefficients of resistance approximately one order of magnitude smaller than the amorphous devices. The desired sensitivity was obtained by initial deposition of an amorphous film on a cold substrate and subsequent vacuum annealing to the desired degree of crystallinity. The characteristics of the device are closely determined by the highest temperature attained during the annealing process [19] .

A diagramatic representation of the detector assem-

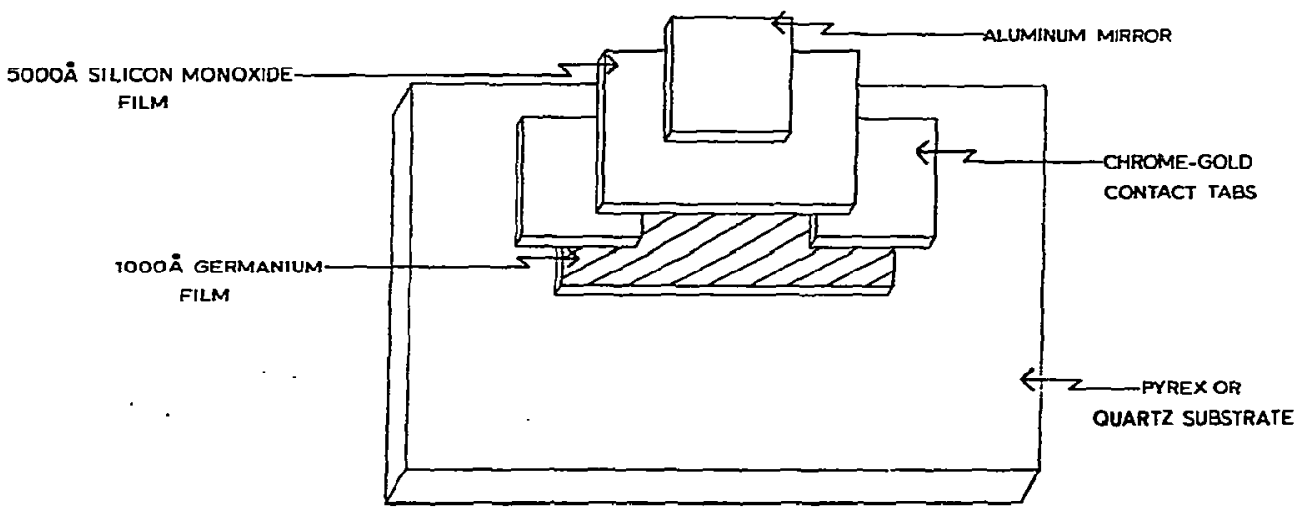

Fig. 1. Schematic illustration of detector assembly. 


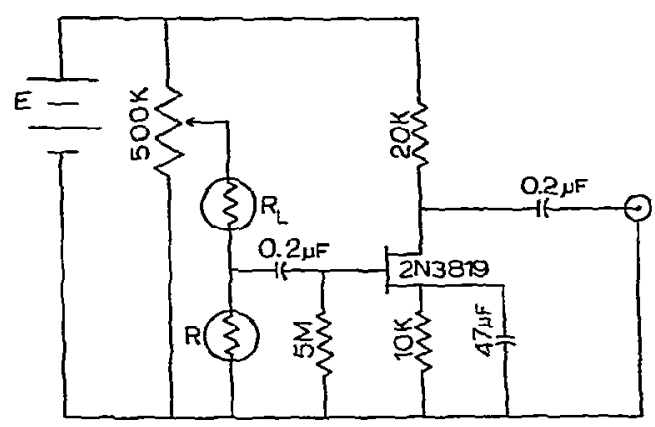

Fig. 2. Detector bridge circuit with low noise impedance matching JFET preamplifier.

bly is shown in fig. 1. The germanium film is first deposited on quartz substrate and the desired electrical characteristics determined by the annealing program described above. Electrical contact is made via evaporated chrome-gold contact tabs. An insulating film of silicon monoxide is deposited over the germanium and contact tab films to prevent electrical contact with the aluminum mirror. The highly reflecting aluminum first surface mirror is used to minimize the direct heating of the germanium film by background radiation and appears to be about $96 \%$ effective in this regard. Detectors are deposited in closely spaced pairs with matched electrical characteristics for use in the compensated voltage divider circuit [20] illustrated in fig. 2 .

\section{Signal analysis}

The absorption of radiation and its degradation to heat causes a power dissipation in the detector consisting of two parts: a constant background $P^{0}$ and an alternating signal component $p_{s}(t)$. In addition to the signal power, the electrical circuit dissipates a power $P_{\mathrm{e}}$ in the detector so that the total power dissipated in the device is $P^{0}+P_{\mathrm{e}}+p_{\mathrm{s}}$. If $P^{0} \ll P_{\mathrm{e}}$, the detector cannot function in a useful manner and we are therefore interested in the analysis for the case where $P^{0} \ll P_{\mathrm{e}}$ can be neglected. letting $C$ and $G$ represent the heat capacity of the detector and the themal conductance between the detector and the bath, respectively, the heat balance equation for the device may be written:

$C \mathrm{~d} T / \mathrm{d} t=P_{\mathrm{e}}+p_{\mathrm{s}}-G \Delta T$, where $\Delta T$ is the temperature rise above ambient bath temperature $\left(T_{0}\right)$. For the case of interest, the incident excitation is chopped at circular frequency $\omega$, so that we may write:

$p_{s}(t)=P_{s}^{0}(1-\cos \omega t) / 2$.

Since the resistance of the detector is a rapidy varying function of temperature [16], the electrical power dissipated in the detector will vary with signal power $p_{\mathrm{s}}(t)$. For the time dependent electrical power, we have that:

$p_{\mathrm{e}}(t)=\left(\partial P_{\mathrm{e}} / \mathrm{\partial} R\right)(\mathrm{d} R / \mathrm{d} T) \Delta T(t)$.

Since:

$P_{\mathrm{e}}=E^{2} R /\left(R_{\mathrm{L}}+R\right)^{2}$,

we may write,

$p_{\mathrm{e}}(t)=\alpha P_{\mathrm{e}}\left(R_{\mathrm{L}}-R\right) \Delta T(t) /\left(R_{\mathrm{L}}+R\right)$,

where we have introduced the detector sensitivity, $\alpha$, defined as:

$\alpha=(1 / R) \mathrm{d} R / \mathrm{d} T$.

It is convenient to introduce at this point two additional detector parameters: the response time $\left(\tau_{D}\right)$ and the responsivity $\mathcal{R}$. These parameters are defined by the following relations:

$\tau_{\mathrm{D}}=\frac{\tau_{\mathrm{T}}}{1-\alpha P_{\mathrm{e}}\left(R_{\mathrm{L}}-R\right) / G\left(R_{\mathrm{L}}-R\right)}$,

$\mathcal{R}=\frac{\alpha e_{\mathrm{D}} R_{\mathrm{L}} /\left(R_{\mathrm{L}}+R\right)}{G-\alpha P_{\mathrm{e}}\left(R_{\mathrm{L}}-R\right) /\left(R_{\mathrm{L}}+R\right)}$.

In the above relations $\tau_{\mathrm{T}}=C / G$ is the usual thermal time constant and $e_{\mathrm{D}}$ is the voltage across the detector. $\mathrm{Eq}$. (1) may now be rewritten in the following form with the aid of eq. (7):

$\mathrm{d} T / \mathrm{d} t=p_{\mathrm{s}}(t) / C-T / \tau_{\mathrm{D}}$.

The time dependence of the temperature fluctuation of the detector may be obtained by integration of eq. (9). Since it is convenient experimentally to utilize phase sensitive detection, the time dependence of the temperature fluctuation is most usefully represented in terms of the in-phase $(\Phi=0)$ and quadrature $(\Phi=90)$ components of the temperature fluctuation with respect to the modulation waveform. The result is given in the equation. 
$\Delta T(\phi)=\frac{P_{\mathrm{s}}^{0} \tau_{\mathrm{D}}\left(\cos \phi+\omega \tau_{\mathrm{D}} \sin \phi\right)}{2 C\left(1+\omega^{2} \tau_{\mathrm{D}}^{2}\right)}$.

The quadrature component is a maximum when $\tau_{D} \omega$ $=1$.

Eq. (10) illustrates several important points regarding the operation of the detector. It is apparent that the detector may be operated in either a positive or negative feedback configuration by the proper choice of $R_{\mathrm{I}}$. Since $\alpha$ for a semiconductor is negative, setting $R_{L}<R$ corresponds to the positive feedback which will produce a correspondingly larger $\Delta T$. However, the response time will increase $\left(\tau_{D}>\tau_{T}\right)$ and the stability of the detector will diminish. With $R_{\mathrm{L}}>R$ we obtain negative feedback and a smaller $\Delta T$, but an improved response time $\left(\tau_{\mathrm{D}}<\tau_{\mathrm{T}}\right)$ and greater stáoility. The responsivity of the detector operated with $R=R_{L}$ has proved adequate for our experimental measurements and under these operating conditions $\tau_{D}=\tau_{T}=$ $C / G$ and $R=\alpha e_{D} / 2 G$.

\section{Noise equivalent power}

The major sources of noise in the detector are electrical Johnson noise $\left(e_{\mathrm{nJ}_{\mathrm{J}}}\right)$ and temperature noise $\left(e_{\mathrm{n}_{\mathrm{T}}}\right)$. The Johnson noise power which results from a finite electrical coupling between the amplifier circuit and the detector element may be estimated with the aid of eq. (8):
$P_{\mathrm{n}_{\mathrm{J}}}=4 G(k T R \Delta f)^{1 / 2} / \alpha e_{\mathrm{D}}$,

where $T$ and $R$ are the operating temperature and resistance of the detector, respectively, and $\Delta f$ is the band width of the amplifier-detector system. The temperature noise power contribution results from a finite thermal coupling between the detector element and the bath through the thermal conductance $G$, and is given by:

$P_{\mathrm{n}_{\mathrm{T}}}=2 T(k G \Delta f)^{1 / 2}$.

The noise equivalent power of the detector may be estimated from eqs. (13) and (14) as:

$\mathrm{NEP}=\left[4 k T G \Delta f\left(T+4 G R / \alpha^{2} e_{\mathrm{D}}^{2}\right)\right]^{1 / 2}$.

The characteristics of a typical detector under two sets of operating conditions are given in table 1 . The values for $C$ and $G$ are crude estimates. The value of $C$, for example, is the heat capacity of the substrate; the effective $C$ will be much less. All other values are experimentaily determined. The thermal time constant of the detector could be improved by at least an order of magnitude by utilizing a saphire substrate of approximately the same mass due to the lower heat capacity of saphire relative to pyrex at cryogenic temperatures. If values of the parameters are compared with those of semiconductor bolometers for infrared detection [21], it will be seen that the NEP and responsivity of our detector is lower by several orders of magnitude, while the thermal response time is several orders of magnitude faster. This results from the trade-off of responsivity

Table 1

Typicaly operating characteristics of the germanium detector

\begin{tabular}{|c|c|c|c|c|c|}
\hline & Parameter & Case I & Case II & Units & Note \\
\hline \multirow[t]{10}{*}{. } & $e_{D}$ & 0.5 & 0.5 & volt & \\
\hline & $R$ & 24.2 & 31.1 & kohm & \\
\hline & $R_{\mathrm{L}}$ & 25 & 30 & kohm & \\
\hline & $c$ & 82 & 5 & rjoule/K & ref. [28] \\
\hline & $T_{0}$ & 5 & 2 & K & \\
\hline & $\alpha$ & -0.1 & -0.25 & $\mathrm{~K}^{-1}$ & \\
\hline & $R$ & 0.18 & & volt/watt & \\
\hline & ${ }^{\tau} \mathrm{D}$ & 30 & 2 & $\mu \mathrm{S}$ & \\
\hline & NEP (calc.) & $1.8 \times 10^{-8}$ & $5.3 \times 10^{-9}$ & watt/Hz $z^{1 / 2}$ & \\
\hline & MEF (obs.) & $\approx 1.0 \times 10^{-9}$ & & watt $/ \mathrm{Hz}^{1 / 2}$ & \\
\hline
\end{tabular}


for response time accomplished by modification of the effective magnitude of the thermal conductance, $G$. Our detector utilized a $G$ of approximately $0.1 \mathrm{Watt} / \mathrm{K}$ which was determined partly by the thermal conductance of the substrate material (pyrex) at cryogenic temperatures [22] and partly by the Kapitza resistance of the bath-detector interface [23]. Infrared detectors are designed to operate with a $G$ of approximately $10^{-4}$ Watt/K.

\section{Thermo-optical spectra}

The magnitude of the thermal power dissipated in solid samples by optical excitation and subsequent radiationless relaxation may be easily estimated from the following equation for the steady state power dissipation in a sample under optical illumination:

$P_{s}^{0}(v)=I^{0}(v) \phi(v) \frac{[1-R(\nu)]\{1-\exp [-k(\nu) x]\}}{1-R(\nu) \exp [-k(\nu) x]}$.

Eq. (14) is appropriate for absorption of normally incident radiation by a parallel sided platelet. $I^{0}(\nu)$ is the illumination intensity at frequency $\nu, R(\nu)$ is the surface reflectivity, $k(\nu)$ is the optical absorption constant, $x$ is the sample thickness and $\phi(\nu)$ is the thermal yield which is defined in terms of the photon quantum yield as:

$\phi(\nu)=\left[1-\nu_{0} \Phi(\nu)\right] / \nu$,

where $\nu_{0}$ is the mean frequency of emission. For small values of $k(v)$, the power dissipation is simply:

$P_{\mathrm{s}}^{0}(\nu) \approx I^{0}(\nu) \phi(\nu) k(\nu) x$.

We note from the form of eq. (16) that the signal developed in the thermo-optical spectrum is directly proportional to the incident intensity. For large values of the sample absorption the power dissipation becomes:

$P_{\mathrm{s}}^{0}(\nu) \approx I^{0}(\nu) \phi(\nu)[1-R(\nu)]$.

Finally, for intermediate values of the absorption the thermo-optical spectrum is a complicated function of sample and instrumental parameters.

The performance of the detector is illustrated in fig. 3 which compares the output of a carbon black coated detector to that of a calibrated type G-3 Eppley thermopile over the wavelength range $2000 \AA$ to $7000 \AA$. The illumination source was as $1 \mathrm{~kW}$ xenon lamp. The

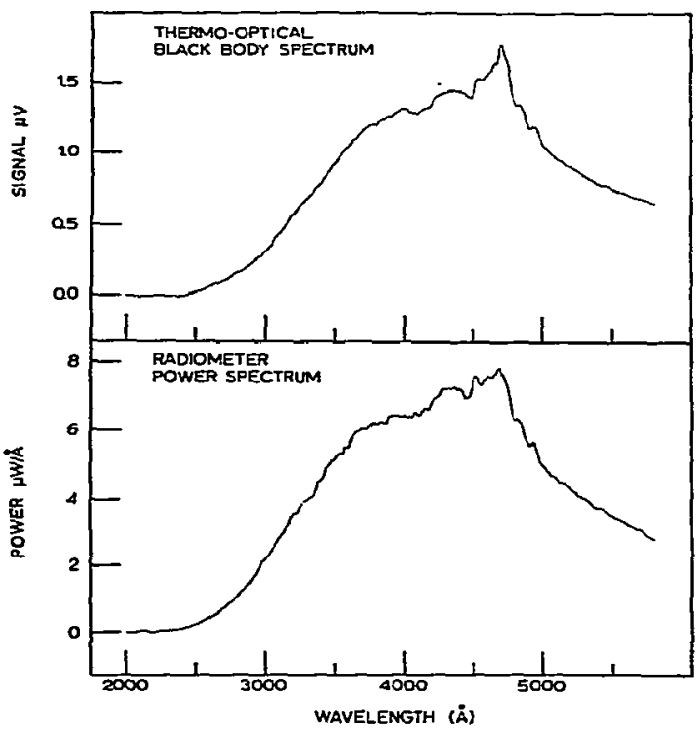

Fig. 3. A comparison of the excitation source power spectrum as recorded by a commercial radiometer and a carbon-black coated detector.

calibration of the thermopile was checked using a chemical actinometer technique based on the photolysis of potassium ferrioxalate [24] and found to be accurate to within $\pm 2 \%$.

Samples for thermo-optical spectra have been prepared by dissolving the material in a volatile solvent and evaporating a drop of the solution on the mirrored surface of the detector. In this manner we were able to produce a relatively uniform and adherent thin film of the sample in contact with the detector surface. It has also been possible to bond small single crystals directly to the detector using silicone oil.

We have recorded thermo-optical spectra by chopping the optical excitation at frequency $\omega$ and monitoring the thermal yield in quadrature with the excitation waveform using phase sensitive detection. An experiment conducted in this fashion yields a flat baseline in regions of no sample absorption and is analogous to an emission excitation experiment utilizing a phosphorescope. In both experiments, the signal observed in regions of sample absorption is convoluted with the intensity distribution of the excitation source and, additionally, a fraction of the signal power is lost by utilizing quadra- 
ture detection to eliminate background signals. Both of these difficulties may be overcome in a more elaborate thermo-optical experiment in which a beam splitter is inserted in the excitation beam. One half of the excitation intensity is allowed to fall on a co-evaporated reference detector coated with carbon black and the other half on the "sample" detector. The total incident intensity is again chopped and the demodulated in-phase signal from the "sample" detector is divided by the demodulated in-phase output from the reference detector. The normalization may be accomplished with either an analog divider circuit or digitally. Presently, we are employing a calculator interface to nor. malize digitally in real time.

\section{Results and discussion}

For our initial experiments biacetyl was used since vapor phase data from opto-acoustic experiments have been published by Kaya et al. [5]. The photophysics of the lower energy excited states of biacetyl vapor may be summanzed as follows [25-27]. Excitation inio the lowest singlet state $\left(S_{1}\right)$ below about $4430 \AA$ is followed by prompt intersystem crossing and relaxation to the lowest triplet $\left(T_{1}\right)$ at about $5000 \AA$. Relaxation of $T_{1}$ to $S_{0}$ is relatively slow (ca. $1 \mathrm{~ms}$ ). At wavelengths shorter than $4430 \mathrm{~A}$ excitation may occur into $T_{2}$ which relaxes rapidly to $S_{0}$ due to a relatively large direct spin orbit matrix element between these states. Excitation into $S_{2}(2700 \AA)$ is followed by rapid photochemical decomposition.

The phosphorescence excitation spectrum of purifred biacetyl and thermo-optical spectrum monitored in-quadrature with the excitation are displayed together in fig. 4 . The very weak band in the thermal excitation spectrum of biacetyl at about $4900 \AA$ is due to direct heating by triplet absorption. These features are evidently not observed in the vapor phase optoacoustic spectra.

The thermo-optical spectrum shown in the center trace was obtained at a chopping frequency of $100 \mathrm{~Hz}$. Major differences between the thermo-optical and optical excitation spectra of biacetyl occur when excitation at a particular wavelength produces an electronic or vibronic state which does not relax exclusively via the lowest triplet state of biacetyl. Therefore, if the thermo-optical and optical excitation spectra are in

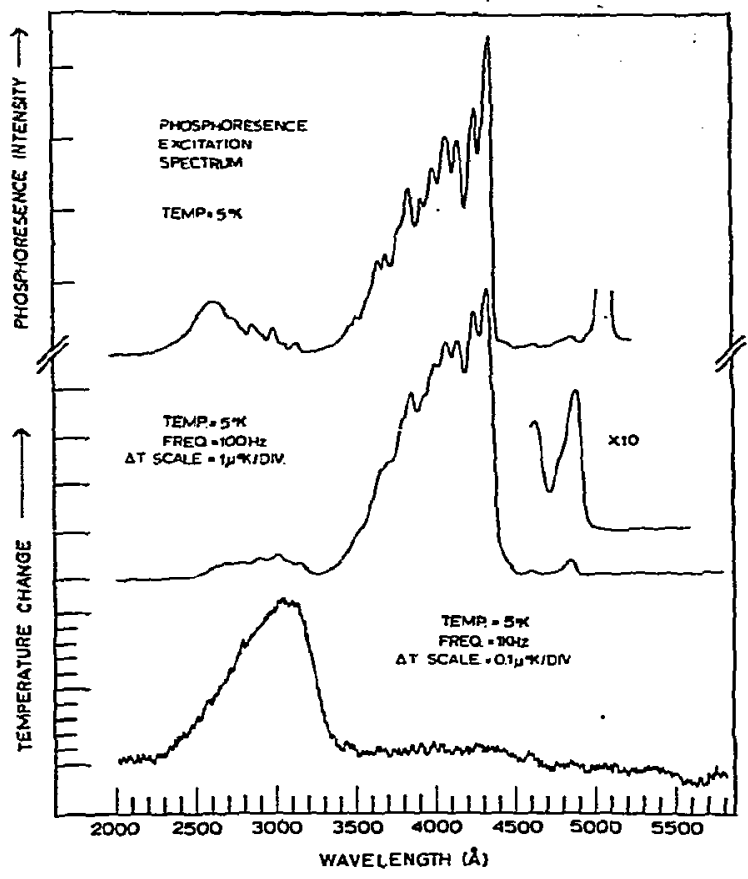

Fig. 4. A comparison of the phosphorescence excitation and therm o-optical spectra of biacetyl.

one-to-one correspondence we may conclude that essentially all optical excitations of the molecule relax via the lowest triplet state. Lack of direct correspondence between the spectra suggests the presence of radiationless pathways for electronic relaxation which do not involve the lowest triplet state of the molecule.

For the most part the thermo-optical and optical excitation spectra of biacetyl are in direct correspondence indicating that essentially all relaxation pathways in crystalline biacetyl at cryogenic temperatures are via the lowest triplet state. The slight enhancement of the thermo-optical signal relative to the phosphorescenceexcitation intensity which is apparent to shorter wavelengths of about $4200 \AA$ may result from $T_{2}$ excitation followed by rapid radiationless decay to $S_{0}[5]$. The major deviation from direct correspondence evident in our data occurs in the region about $3100 \mathrm{~A}$. If the uppermost and center spectra of fig. 4 are compared, an enhancement of the themo-optical signal relative to the optical excitation signal is apparent at $3100 \AA$.

The phosphorescence lifetime of crystalline biacetyl 
at liquid helium temperature is reported to be $2.5 \mathrm{~ms}$ [27]. The response time of the detector is, therefore, several orders of magnitude faster than the triplet decay time, and the thermal time constant in eq. (10) may be replaced by the triplet lifetime. By increasing the modulation frequency of the excitation source to $1 \mathrm{kHz}$ it is possible to attenuate the signal derived from heating due to triplet non-radiative relaxation processes. From eq. (10) with the thermal time constant replaced by the triplet lifetime of biacetyl, the signal from the non-radiative relaxation of the triplet state of biacetyl should be reduced by a factor of about twenty five when the chopping frequency is raised from 100 $\mathrm{Hz}$ to $1 \mathrm{kHz}$. When this is done, the thermo-optical spectrum shown in the lower most trace of fig. 4 is obtained indicating the excitation in a rather narrow spectral region about $3100 \AA$ produces a fast radiationless relaxation process probably associated with $S_{2}$ excitation and subsequent rapid photo-chemical decomposition.

Our experimental results are in qualitative agreement with those obtained by Kaya et al. [5] from vapor phase opto-acoustic spectral measurements. In view of the substantial differences in radiationless relaxation processes in vapor and condensed phases, the general overall agreement between the two sets of data was somewhat unanticipated.

We have also recorded the thermo-optical spectra of

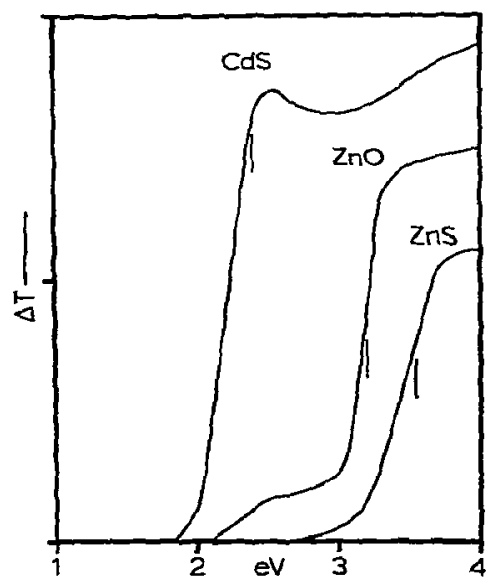

Fig. 5. The thermo-optical spectra of several semiconductors in the region of their band-gap absorption. several materials whose absorption spectra are not directly available. In fig. 5 we illustrate the thermo-optical spectra of several semiconductors in the region of their band gaps. These materials were obtained as fine powders to which we added a small amount of mineral oil to serve as a binding agent. The surface of the detector was then painted with a thin coating of the material and the thermo-optical spectrum recorded in quadrature with the excitation illumination. These spectra may be compared with several of those obtained by Rosencwaig [3] using the opto-acoustic technique on room temperature samples. Many of the details of the spectra of these materials which could be observed at low temperatures in high resolution spectra are obscured by the wide excitation slits used to obtain the spectra. The use of tunable laser excitation with thermo-optical detection at liquid helium temperatures, however, will certainly provide considerable enhancement of the details of the structures in these spectra and that of similar materials. These experiment are currently in progress.

\section{Conclusion}

The principal advantage of this technique involves measurements of the optical absorption spectra of thin films on surfaces and polycrystalline materials. The low temperatures employed provide excellent resolution of the spectral features and the nature of the detector is such as to provide extremely good signal to noise with only moderate precautions. It has been our observation that the source or detector noise is below the preamplifier noise for the lowest noise preamplifiers commercially available. The technique is quite sensitive and easily measures absorption in thin films of materials on the order of $1000 \AA$ in thickness.

A second distinguishing characteristic of the thermooptical method is its ability to detect and measure very weak absorption bands such as those arising from spin and/or symmetry forbidden transitions or trace impurities. Excitation experiments, in general, have an advantage over absorption experiments in this regard due to the fact that the signal/noise ratio may be improved by simply increasing the intensity of the excitation source. The intensity available from narrow band tunable lasers makes the excitation experiment particularly attractive in this regard. 


\section{Acknowledgement}

The authors would like to acknowledge partial support for this work from the Air Force Materials Laboratory. One of us (K.W.H.) would like to express his appreciation to the National Science Foundation for support during his tenure as an Energy Related Postdoctoral Fellow.

\section{References}

[1] W.R. Harshbarger and M.B. Robin, Accounts Chem. Res. 6 (1973) 329.

[2] W.R. Harshbarger and M.B. Robin, Chem. Phys. Letters 21 (1973) 362.

[3] A. Rosencwaig, Phys. Today 23 (1975).

[4] A.G. Bell, Proc. Am. Assoc. Acad. Sci. 29 (1880) 115.

[5] K. Kaya, W.R. Harsh barger and M.B. Robin, J. Chem. Phys. 60 (1974) 4231.

[6] A.W. Read, Advan. Mol. Relaxation Processes 1 (196768) 275 .

[7] M.R. Robin, Plenary Lecture, International Spectroscopy Symposium, Ohio State University (1974).

[8] H. Parker and A.H. Francis, Contributed Paper, International Molecular Spectroscopy Symposium, Ohio State University (1974).

[9] J.T. Houghton and S.D. Smith, Infrared physics (Oxford Univ. Press, London, 1966).
[10] W.S. Boyle and K.F. Rodgers Jr., J. Opt. Soc. Am. 49 (1959) 66.

[11] C.E. Jones et al., Appl. Opt. 4 (1965) 683.

[12] V. Narayanamurti and R.C. Dynes, Phys. Rev. Letters 28 (1972) 1461.

[13] W.F. Giauque, J.W. Stout and C.W. Clark, J. Am. Chem. Soc. 60 (1938) 1053.

[14] D.E. Bradley, Brit. J. Appl. Phys. 5 (1954) 65.

[15] C. Terry, Rev. Sci. Instr. 39 (1976) 925.

[16] A.H. Clark, Phys. Rev. 154 (1976) 750.

[17] Y. Sakai and K. Takahashi, Japan. J. Appl. Phys. 2 (1963) 629.

[18] H. Ehrenreich et al. Fundamentals of Amorphous Semiconductors, Natl. Acad. Sci., Washington, D.C. (1972).

[19] P.A. Walley and A.K. Jonscher, Thin Solid Films 1 (1967) 367.

[20] L.C. Robinson, Experimental physics, Vol. 10 (Academic Press, New York, 1973) p. 169.

[21] F.J. Low, J. Opt. Soc. Am. 51 (1961) 1300.

[22] R.L. Powell and W.A. Blanpied, National Bureau of Standard Circular No. 556 (1954) p. 68.

[23] G.L. Pollack, Rev. Mod. Phys. 41 (1960) 48.

[24] C.G. Hatchard and C.A. Parker, Proc. Roy. Soc. 235 (1956) 518.

[25] H.W. Sidebottom et al., J. Am. Chem. Soc. 94 (1972) 13.

[26] E. Drent, R.P. van der Werf and J. Kommandeur, J. Chem. Phys. 59 (1973) 2061.

[27] J.C.D. Brand and A.H.W. Miau, J. Am. Chem. Soc. 96 (1974) 4380.

[28] P.L. Smith and N.M. Wolcott, Phil. Mag. 1 (1956) 854. 